\title{
PREVENÇÃO E TRATAMENTO DE OBESIDADE E DISLIPIDEMIA EM CRIANÇAS E ADOLESCENTES: UMA REVISÃO INTEGRATIVA
}

\author{
PREVENTION AND TREATMENT OF OBESITY AND DYSLIPIDEMIA IN CHILDREN AND \\ ADOLESCENTS: AN INTEGRATIVE REVIEW
}

DOI: $10.16891 / 2317-434 X . v 8 . e 2 . a 2020 . p p 591-602$

Recebido em: 25.05.2020 | Aceito em: 16.07.2020

\begin{abstract}
Nicolly Castelo Branco Chaves*a, Lucas Farias Lopesa, Maria Andrezza Gomes Maiaa, Vinícius

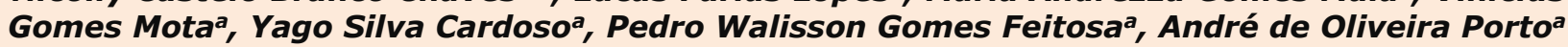

Faculdade de Medicina da Universidade Federal do Cariria E-mail: nicollycastelo10@gmail.com
\end{abstract}

\section{RESUMO}

A obesidade e a dislipidemia são preocupações crescentes na população infanto-juvenil. Este artigo objetiva realizar uma revisão integrativa da literatura sobre os aspectos multifatoriais da prevenção e do tratamento da obesidade e da dislipidemia na infância e adolescência, considerando as relações fisiopatológicas e metabólicas envolvidas nesse distúrbio. Foi realizada uma revisão integrativa da literatura no banco de dados eletrônicos PubMed e na plataforma BVS. Os artigos foram selecionados, entre 2015 e 2020, utilizaram as palavras-chave "obesidade"; "dislipidemia"; "infância" e "adolescente", baseando-se no idioma e no período de publicação, bem como inadequação aos seguintes tópicos, como critérios de exclusão: metodologia e objetivo da revisão. Inseridas as palavras-chave, foram selecionados 1503 estudos que, passados por 3 etapas de filtragem, resultaram em 10 artigos para síntese qualitativa. Observou-se que crianças e adolescentes obesas tendem a ter mais dislipidemia. É evidenciada uma relação significativa entre obesidade, sobrepeso, circunferência abdominal e triglicerídeos alterados. A adoção de dietas com ômega-3 e leite enriquecido com esteróis vegetais apresentaram efeitos benéficos no tratamento da obesidade. Ademais, o uso de atorvastatina também apresentou resultados positivos em crianças e adolescentes com hipercolesterolemia familiar. Foi observado também que o incentivo familiar influencia na continuidade do tratamento. Concluiu-se que a obesidade e a dislipidemia sofrem significativas influências do meio, como dieta e ausência de atividade física. Apesar dos benefícios encontrados da associação de mudança de estilo de vida e uso de terapia medicamentosa, fazem-se necessários mais estudos para análise dessa associação terapêutica a longo prazo.

Palavras-chave: obesidade; dislipidemia; infância; adolescente

\section{ABSTRACT}

Obesity and dyslipidemia are growing concerns in children and adolescents. This article aims to carry out an integrative literature review on the multifactorial aspects of the prevention and treatment of obesity and dyslipidemia in childhood and adolescence, considering the pathophysiological and metabolic relationships involved in this disorder. A integrative literature review was carried out in the electronic database PuBMed and in the BVS platform. The articles were selected, between 2015 and 2020, used as keywords "obesity"; "dyslipidemia"; "childhood" and "adolescent", based on the language and period of publication, as well as the inadequacy to the following topics, such as the exclusion criterion: methodology and purpose of the review. Inserted as keywords, 1503 studies were selected which, after 3 filtering steps, resulted in 10 articles for qualitative demonstration. As for the results, it was observed that obese children and adolescents tend to have more dyslipidemia. A significant relationship between obesity, overweight, waist circumference and altered triglycerides is evidenced. The adoption of ômega-3 diets and milk enriched with plant sterols had beneficial effects in the treatment of obesity. In addition, the use of atorvastatin has also shown positive results in children and adolescents with familial hypercholesterolemia. It was also observed that Family incentives influence the continuity of treatment. It was concluded that obesity and dyslipidemia suffer significant influences from the environment, such as diet and physical activity. Despite the benefits found in the association of lifestyle change and the use of drug therapy, further studies are needed to analyze this therapeutic association in the long term.

Keyword: obesity; dyslipidemia; childhood; teenager. 


\section{INTRODUÇÃO}

A obesidade é definida como acúmulo excessivo de gordura corpórea (OLIVEIRA et al., 2020). Foi desenvolvida uma classificação para diagnóstico, segundo a Endocrine Society, em que: sobrepeso é dado por IMC maior ou igual a $85 \%$; obesidade é considerada acima de $95 \%$ e obesidade extrema a partir de $120 \%$ (IZAR et al., 2019). A resistência à insulina, a tolerância reduzida à glicose, a dislipidemia e a hipertensão são os reflexos metabólicos mais frequentes da obesidade (PASTORVILLAESCUSA et al., 2016). A dislipidemia caracteriza-se, principalmente, pelos níveis elevados de triglicerídeos e de proteínas de baixa densidade (LDL) no plasma sanguíneo, juntamente à redução das proteínas de elevada densidade (HDL) (HIGGINS; ADELI, 2019).

No contexto pediátrico, o sobrepeso e a obesidade geram maior atenção, visto que houve crescimento de suas prevalências em crianças e adolescentes a nível mundial. Os ganhos acentuados nos índices de massa corpórea (IMC) nos primeiros meses de vida são considerados preditores desse distúrbio metabólico ao longo da infância e adolescência, tornando imprescindível a prevenção e o tratamento precoce da obesidade (CAMARGOS et al., 2019). A prevalência desse distúrbio metabólico nas fases iniciais da vida é observada em diferentes países. No México, a atualização da Pesquisa Nacional de Saúde e Nutrição, realizada em 2016, revela obesidade e sobrepeso prevalentes entre adolescentes: $39,2 \%$ e $33,5 \%$ para o sexo feminino e masculino, respectivamente (DELRÍO-NAVARRO et al., 2019).

A preocupação global do desenvolvimento da obesidade e suas consequentes alterações metabólicas, como a dislipidemia, está associada às interferências desses distúrbios em longo prazo sobre a saúde dos jovens e adultos. Lind et al., (2019) refere que os hábitos alimentares desenvolvidos durante a infância exercem efeitos duradouros sobre a saúde humana. Diante desse contexto, a dislipidemia na infância e adolescência e sua alta prevalência são importantes preditores da aterosclerose na fase adulta, tornando-se foco essencial de prevenção e tratamento (REUTER et al., 2018).

Ademais, o desenvolvimento de aterosclerose em crianças e adolescentes com obesidade apresenta uma interface ainda mais preocupante quanto aos impactos desse distúrbio metabólico no risco cardiovascular (GIDDING et al., 2015). A disfunção endotelial e rigidez arterial características da aterosclerose geram, em longo prazo, problemas cardíacos e vasculares. Tendo em vista isso, desenvolve-se uma reflexão sobre a construção multifatorial da obesidade infantil, associada, principalmente, ao estilo de vida sedentário e a mudança no padrão alimentar da sociedade atual, caracterizado pelo aumento do consumo de fast-food, alimentos industrializados com elevados índices de açúcar, sal e gordura (IZAR et al., 2019). Dessa maneira, faz-se importante o estudo sobre formas de prevenção da obesidade na infância, visto que esse distúrbio está associado a sérios riscos de saúde, como esteatose hepática, doenças coronarianas, indução de mecanismos inflamatórios, além dos distúrbios metabólicos associados à Diabetes Tipo II (YUCA et al., 2016).

A partir de uma breve exposição sobre o panorama da obesidade e da dislipidemia entre crianças e adolescentes, torna-se evidente que esta é uma problemática mundial de saúde pública. Assim, este estudo busca realizar uma revisão integrativa da literatura quanto aos aspectos multifatoriais acerca da prevenção e tratamento dos distúrbios metabólicos supracitados, considerando-se seus impactos a longo prazo sobre o bem-estar de crianças e adolescentes.

\section{METODOLOGIA}

Foi realizada uma busca nas bases de dados eletrônicos PubMed e BVS para selecionar estudos publicados sobre os aspectos multifatoriais associados à prevenção e tratamento da obesidade e da dislipidemia em indivíduos na infância e adolescência, apresentando recortes epidemiológicos, manifestações clínicas e laboratoriais e relações farmacológicas. Havia dois revisores independentes e um terceiro revisor consultado em casos de artigos de interesse conflitante.

Foram aplicadas as palavras-chave "Obesity"; dyslipidemia"; "children" e "teenager" para identificar artigos publicados entre janeiro de 2015 e março de 2020. Os critérios de inclusão foram estudos publicados em inglês, adequação ao objetivo desta revisão, rigor metodológico empregado e artigos disponíveis gratuitamente na íntegra. Foram excluídos artigos de revisão, bem como comentários de literatura, editoriais, comunicações e cartas ao editor. Cada artigo foi lido na íntegra e suas informações foram dispostas em uma planilha no programa Microsoft Excel, incluindo ano de publicação, autores, base de dados, periódico, país de realização do estudo, objetivo, metodologia, resultados e conclusão. Posteriormente, os principais achados de cada artigo selecionado na amostra final foram compilados nesta revisão, sendo discutidos consoante a literatura.

\section{RESULTADOS}

Foram recuperados um total de 1.503 artigos 
utilizando a estratégia de busca. Após realizar triagem baseada por idioma e por período de publicação (janeiro de 2015 a março de 2020) - foram excluídos 867 estudos, restando 636 estudos. Após esta etapa de busca, 621 artigos foram excluídos por não tratarem do objetivo desta revisão designado na estratégia de busca, permanecendo 15 artigos para avaliação em texto completo. Desses artigos, 5 foram excluídos por inadequação metodológica. Assim, 10 estudos foram incluídos para síntese qualitativa (Figura 1). Destes, 8 dos trabalhos selecionados foram retirados da base de dados PubMed e 2 da plataforma BVS, sendo indexados em LILACS. As principais características dos estudos incluídos são apresentadas no Quadro 1.

Figura 1. Estratégia de busca eletrônica por estudos.
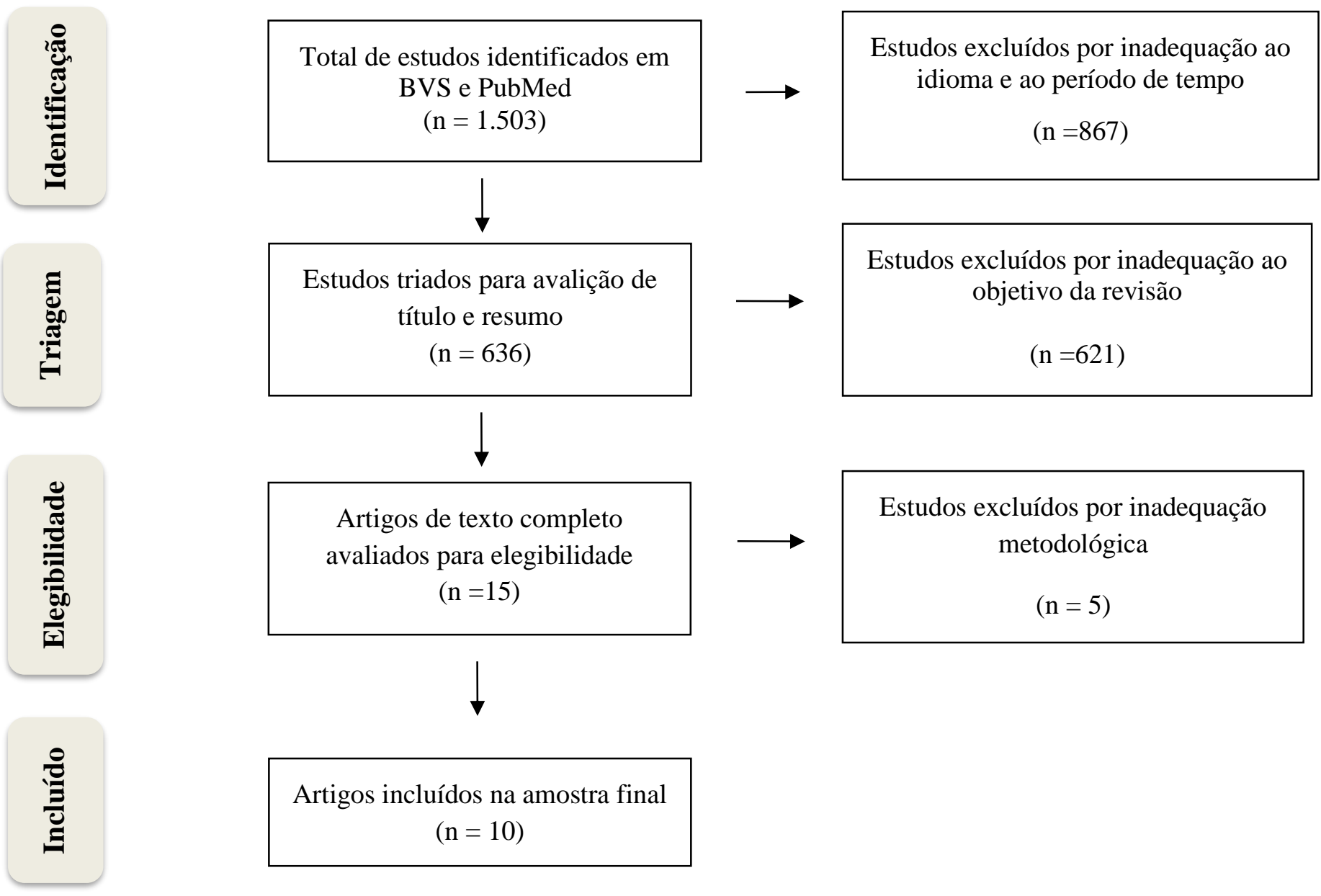

Estudos excluídos por inadequação ao objetivo da revisão

$$
(\mathrm{n}=621)
$$

Estudos excluídos por inadequação metodológica

$$
(\mathrm{n}=5)
$$




\section{-

Quadro 1. Síntese da amostra final de estudos selecionados.

\begin{tabular}{|c|c|c|c|c|c|}
\hline AUTOR / ANO & PAÍS & OBJETIVO & METODOLOGIA & RESULTADOS & CONCLUSÃO \\
\hline $\begin{array}{l}\text { ALMEIDA } \\
\text { et al., } 2016\end{array}$ & Brasil & $\begin{array}{l}\text { O estudo objetiva } \\
\text { determinar o perfil de } \\
\text { sobrepeso e de lipídios } \\
\text { de crianças na faixa } \\
\text { etária de } 6 \text { a } 9 \text { anos. }\end{array}$ & $\begin{array}{l}\text { O grupo teste foi } \\
\text { constituído por } \\
\text { crianças de } 6 \text { a } 9 \text { anos } \\
\text { de escolas públicas } \\
\text { de Vitória-ES. Foram } \\
\text { coletados amostras de } \\
\text { sangue, além de } \\
\text { aferir o peso, a altura } \\
\text { e a circunferência } \\
\text { abdominal. }\end{array}$ & $\begin{array}{l}\text { Avaliação nutricional } \\
\text { mostrou estatísticas de } \\
\text { sobrepeso e obesidade } \\
\text { alarmantes. A análise } \\
\text { das tabelas, } \\
\text { com variáveis } \\
\text { antropométricas e } \\
\text { dosagens lipídicas } \\
\text { mostrou significante } \\
\text { relação entre } \\
\text { obesidade, sobrepeso, } \\
\text { circunferência } \\
\text { abdominal alterada e } \\
\text { triglicerídeos } \\
\text { alterados. }\end{array}$ & $\begin{array}{l}\text { Identificou-se que o } \\
\text { aumento de triglicerídeos } \\
\text { esteve associado ao } \\
\text { aumento do IMC, e da } \\
\text { circunferência abdominal. } \\
\text { Houve também prevalência } \\
\text { de sobrepeso e de } \\
\text { obesidade em altos índices, } \\
\text { considerando a faixa etária } \\
\text { pediátrica. }\end{array}$ \\
\hline $\begin{array}{l}\text { DEL-RÍO- } \\
\text { NAVARRO } \\
\text { et al., } 2019\end{array}$ & México & $\begin{array}{l}\text { O estudo tem como } \\
\text { objetivo avaliar a } \\
\text { eficácia e segurança da } \\
\text { suplementação de ácidos } \\
\text { graxos ômega } 3 \text { em } 130 \\
\text { pacientes crianças e } \\
\text { adolescentes, entre } 10 \text { e } \\
16 \text { anos, com IMC } 95 \text { e } \\
\text { níveis séricos de } \\
\text { triglicérideos variando } \\
\text { de } 150 \text { a } 1000 \mathrm{mg} / \mathrm{dL} .\end{array}$ & $\begin{array}{l}\text { Foi realizado um } \\
\text { ensaio clínico duplo- } \\
\text { cego e randomizado } \\
\text { controlado por } \\
\text { placebo. }\end{array}$ & $\begin{array}{l}\text { Houve uma maior } \\
\text { redução nas } \\
\text { concentrações de } \\
\text { triglicerídeos no grupo } \\
\text { tratado com } 3 \mathrm{~g} / \text { dia de } \\
\text { ômega } 3 \text {, em relação } \\
\text { ao tratado com } \\
\text { placebo. }\end{array}$ & $\begin{array}{l}\text { A suplementação com } \\
\text { ácidos graxos ômega } 3 \\
\text { apresentaram-se seguros e } \\
\text { eficazes na redução das } \\
\text { concentrações séricas de } \\
\text { triglicerídeos em crianças } \\
\text { com obesidade e } \\
\text { hipertrigliceridemia. }\end{array}$ \\
\hline $\begin{array}{l}\text { EDBERG et } \\
\text { al., } 2019\end{array}$ & EUA & $\begin{array}{l}\text { Testar a eficácia de } \\
\text { concentrar incentivos em } \\
590 \text { jovens, entre } 5 \text { e } 19 \\
\text { anos, participantes do } \\
\text { Programa de bem-estar } \\
\text { cardíaco Healthy Hearts, } \\
\text { administrado pela prática } \\
\text { de cardiologia pediátrica } \\
\text { do Children's Heart } \\
\text { Center (CHC) - Nevada/ } \\
\text { Estados Unidos, para } \\
\text { redução do IMC ou } \\
\text { divisão os incentivos } \\
\text { com seus pais. }\end{array}$ & $\begin{array}{l}\text { Dois grupos, um de } \\
\text { incentivo focado e } \\
\text { outro de incentivo } \\
\text { partilhado com os } \\
\text { pais, participaram de } \\
\text { atividades físicas, } \\
\text { educação nutricional } \\
\text { e discussão de } \\
\text { estratégias positivas } \\
\text { para implementar } \\
\text { mudança no estilo de } \\
\text { vida. }\end{array}$ & $\begin{array}{l}\text { Os jovens designados } \\
\text { para o grupo de } \\
\text { incentivos focados } \\
\text { obtiveram uma } \\
\text { redução significativa } \\
\text { do IMC durante o } \\
\text { primeiro trimestre da } \\
\text { pesquisa; } \\
\text { posteriormente o outro } \\
\text { grupo conseguiu } \\
\text { redução semelhante. }\end{array}$ & $\begin{array}{l}\text { A análise dos dados do } \\
\text { comportamento } \\
\text { experimental do estudo } \\
\text { indica que o impacto } \\
\text { inicial da estrutura de } \\
\text { incentivos é significativo, } \\
\text { mas esse impacto se } \\
\text { dissipa com o tempo. }\end{array}$ \\
\hline INGE et al., & EUA & Analisar os estudos a & Foi realizada uma & Desde o início até o & A Cirurgia resultou em \\
\hline
\end{tabular}




\begin{tabular}{|c|c|c|c|c|c|}
\hline 2017 & & $\begin{array}{l}\text { longo prazo da ponte } \\
\text { gástrica em Y de Roux } \\
\text { em uma coorte de jovens } \\
\text { submetidos a cirurgia } \\
\text { bariátrica na } \\
\text { adolescência. }\end{array}$ & $\begin{array}{l}\text { análise prospectiva } \\
\text { de acompanhamento } \\
\text { dos resultados desses } \\
\text { pacientes } 5 \text { a } 12 \text { anos } \\
\text { após a cirurgia. } \\
\text { Avaliou-se o IMC, } \\
\text { comorbidades, status } \\
\text { de micronutrientes e } \\
\text { segurança. }\end{array}$ & $\begin{array}{l}\text { longo prazo, foram } \\
\text { registrados declínios } \\
\text { significativos de } \\
\text { pressão arterial } \\
\text { elevada, dislipidemia e } \\
\text { de diabetes tipo } 2 \text {. }\end{array}$ & $\begin{array}{l}\text { redução do peso corporal, } \\
\text { além de benefícios } \\
\text { cardiometabólicos para os } \\
\text { jovens. }\end{array}$ \\
\hline $\begin{array}{l}\text { LANGSLET et } \\
\text { al., } 2016\end{array}$ & Noruega & $\begin{array}{l}\text { Caracterizar a eficácia e } \\
\text { a segurança da } \\
\text { atorvastatina por mais de } \\
3 \text { anos e avaliar o } \\
\text { impacto no crescimento } \\
\text { e no desenvolvimento de } \\
\text { crianças com } \\
\text { hipercolesterolemia } \\
\text { familiar (HeFH). }\end{array}$ & $\begin{array}{l}\text { Foi administrada a } \\
\text { atorvastatina aos } \\
\text { indivíduos com base, } \\
\text { mas medidas do } \\
\text { LDL-C. }\end{array}$ & $\begin{array}{l}\text { As reduções médias } \\
\text { do LDL-C foram } \\
\text { significativamente } \\
\text { altas. A atorvastatina } \\
\text { teve um perfil } \\
\text { favorável de segurança } \\
\text { e de tolerabilidade. }\end{array}$ & $\begin{array}{l}\text { O tratamento com } \\
\text { atorvastatina por mais de } 3 \\
\text { anos mostrou-se eficaz e } \\
\text { não teve impacto no } \\
\text { crescimento/maturação de } \\
\text { crianças e de adolescentes } \\
\text { com HeFH. Alguns } \\
\text { eventos adversos foram } \\
\text { observados. }\end{array}$ \\
\hline $\begin{array}{l}\text { LEVERSON et } \\
\text { al., } 2017\end{array}$ & EUA & $\begin{array}{l}\text { Examinar os efeitos da } \\
\text { intervenção nutricional } \\
\text { intensiva nos níveis de } \\
\text { PCSK9 em adolescentes } \\
\text { com sobrepeso e com } \\
\text { fatores de risco para } \\
\text { doença cardiovascular. }\end{array}$ & $\begin{array}{l}\text { Foram entregues } \\
\text { refeições baseadas } \\
\text { em dieta de baixa } \\
\text { gordura e baixa carga } \\
\text { glicêmica nas casas } \\
\text { de todos os } \\
\text { participantes. As } \\
\text { medidas } \\
\text { antropométricas, os } \\
\text { dados laborais e os } \\
\text { níveis séricos de } \\
\text { proteína PCSK9 } \\
\text { foram aferidos } \\
\text { durante o período. }\end{array}$ & $\begin{array}{l}\text { A alteração no PCSK9 } \\
\text { foi associada a } \\
\text { alteração de insulina } \\
\text { em jejum, insulina } \\
\text { HOMA-IR e AUC, } \\
\text { independentemente da } \\
\text { perda de peso. }\end{array}$ & $\begin{array}{l}\text { PCSK9 diminuiu em } \\
\text { jovens participantes da } \\
\text { dieta intensiva. A alteração } \\
\text { no HOMA-IR foi } \\
\text { associada a alteração no } \\
\text { PCSK9, independente da } \\
\text { perda de peso, fator que } \\
\text { sugere uma importante } \\
\text { relação com a } \\
\text { sensibilidade à insulina }\end{array}$ \\
\hline $\begin{array}{l}\text { NIELSEN } \\
\text { Et al., } 2017\end{array}$ & $\begin{array}{c}\text { Dinamarc } \\
\mathrm{a}\end{array}$ & $\begin{array}{l}\text { Identificar e comparar as } \\
\text { concentrações } \\
\text { plasmáticas lipídicas de } \\
\text { crianças e adolescentes } \\
\text { em jejum, com peso } \\
\text { normal e com } \\
\text { sobrepeso/obesidade. }\end{array}$ & $\begin{array}{l}2.141 \text { crianças da } \\
\text { população de base do } \\
\text { coorte foram } \\
\text { fenotipadas, a partir } \\
\text { da idade, da altura, } \\
\text { do peso, estágio } \\
\text { puberal e de amostras } \\
\text { sanguíneas. } 1.421 \\
\text { crianças e } \\
\text { adolescentes com } \\
\text { IMC acima do } \\
\text { percentil } 90 \text { foram }\end{array}$ & $\begin{array}{l}\text { Em relação ao LDL e } \\
\text { ao colesterol não- } \\
\text { HDL, esses estiveram } \\
\text { mais elevados } \\
\text { significativamente, em } \\
\text { ambos os sexos no } \\
\text { coorte TCOC e em } \\
\text { todas as faixas etárias } \\
\text { analisadas, em relação } \\
\text { à população de base. } \\
\text { A concentração } \\
\text { plasmática de HDL foi }\end{array}$ & $\begin{array}{l}\text { Crianças e adolescentes } \\
\text { com obesidade exibiram, } \\
\text { no geral, concentrações } \\
\text { mais altas de Colesterol } \\
\text { total, de LDL, de não-HDL } \\
\text { e de triglicerídeos, e } \\
\text { concentrações mais baixas } \\
\text { de HDL do que na } \\
\text { população de base, } \\
\text { demonstrando que crianças } \\
\text { e adolescentes da } \\
\text { população obesa tendem a }\end{array}$ \\
\hline
\end{tabular}




\begin{tabular}{|c|c|c|c|c|c|}
\hline & & & $\begin{array}{l}\text { incluídas, a partir de } \\
\text { um coorte } \\
\text { previamente } \\
\text { realizado (Coorte } \\
\text { TCOC), as quais } \\
\text { também foram } \\
\text { fenotipadas. }\end{array}$ & $\begin{array}{l}\text { significativamente } \\
\text { mais baixa no coorte } \\
\text { TCOC, do que na } \\
\text { população de base, em } \\
\text { ambos os sexos, em } \\
\text { todas as faixas etárias } \\
\text { analisadas. A } \\
\text { concentração de } \\
\text { colesterol total foi } \\
\text { maior nas garotas, } \\
\text { apenas no grupo mais } \\
\text { velho, e, nos garotos, } \\
\text { na faixa etária } \\
\text { intermediária e na } \\
\text { mais velha. As } \\
\text { concentrações de } \\
\text { triglicerídeos entre } \\
\text { garotos e garotas do } \\
\text { coorte TCOC foram } \\
\text { significativamente } \\
\text { mais altas do que na } \\
\text { população de base. }\end{array}$ & ter mais dislipidemia. \\
\hline $\begin{array}{l}\text { OLIVARES et } \\
\text { al., } 2015\end{array}$ & Espanha & $\begin{array}{l}\text { Determinar a eficácia de } \\
\text { um leite enriquecido } \\
\text { com fitoesteróis para } \\
\text { reduzir a } \\
\text { hipertrigliceridemia em } \\
\text { crianças }\end{array}$ & $\begin{array}{l}\text { Foram administrados } \\
\text { dois tipos de leite: } \\
\text { um placebo e outro } \\
\text { enriquecido com } \\
\text { esteróis vegetais } \\
\text { diluídos. Do início ao } \\
\text { fim do período foram } \\
\text { coletados amostras de } \\
\text { sangue. }\end{array}$ & $\begin{array}{l}\text { O efeito atribuído a } \\
\text { ingestão do leite } \\
\text { enriquecido foi uma } \\
\text { redução tênue nos } \\
\text { níveis de triglicerídeos } \\
\text { basais. }\end{array}$ & $\begin{array}{l}\text { O leite enriquecido com } \\
\text { esteróis vegetais pode } \\
\text { constituir uma estratégia } \\
\text { adequada para o } \\
\text { tratamento da } \\
\text { hipertrigliceridemia } \\
\text { pediátrica. }\end{array}$ \\
\hline $\begin{array}{l}\text { REUTER } \\
\text { et al., } 2019\end{array}$ & Brasil & $\begin{array}{l}\text { Verificar possíveis } \\
\text { relações entre } \\
\text { dislipidemia, fatores } \\
\text { culturais e aptidão } \\
\text { cardiorrespiratória em } \\
\text { escolas. }\end{array}$ & $\begin{array}{l}\text { Foram realizadas } \\
\text { coletas de sangue e } \\
\text { utilizadas amostras } \\
\text { de soro para dosagem } \\
\text { de triglicerídeos e de } \\
\text { colesterol total. Os } \\
\text { níveis de aptidão } \\
\text { cardiorrespiratória } \\
\text { (APCR) foram } \\
\text { avaliados por meio } \\
\text { de testes de corrida. } \\
\text { Uma avaliação }\end{array}$ & $\begin{array}{l}\text { A dislipidemia esteve } \\
\text { associada } \\
\text { significativamente } \\
\text { com o sexo feminino, } \\
\text { com sobrepeso e com } \\
\text { obesidade. } \\
\text { Adolescentes } \\
\text { apresentaram menores } \\
\text { chances de } \\
\text { triglicerídeos e } \\
\text { colesterol total } \\
\text { aumentados do que }\end{array}$ & $\begin{array}{l}\text { Os resultados mostraram a } \\
\text { elevada prevalência de } \\
\text { dislipidemia em escolares } \\
\text { e sua relação com baixa } \\
\text { APCR e fatores culturais, } \\
\text { relacionados ao } \\
\text { comportamento sedentário. }\end{array}$ \\
\hline
\end{tabular}




\begin{tabular}{|c|c|c|c|c|c|}
\hline & & & $\begin{array}{l}\text { cultural foi realizada } \\
\text { por meio de } \\
\text { questionário. }\end{array}$ & crianças. & \\
\hline $\begin{array}{l}\text { YUCA et al., } \\
2016\end{array}$ & EUA & $\begin{array}{l}\text { Estimar as concentrações } \\
\text { séricas de MIC- } \\
\text { 1/GDF15 em crianças } \\
\text { obesas e sua relação com } \\
\text { parâmetros de } \\
\text { obesidade, inflamação, } \\
\text { metabolismo e glicose. }\end{array}$ & $\begin{array}{l}\text { Aferiu-se a } \\
\text { concentração de } \\
\text { MIC-1/GDF15 em } \\
\text { crianças e avaliou-se } \\
\text { sua relação com } \\
\text { parâmetros } \\
\text { antropométricos, } \\
\text { bioquímicos e } \\
\text { ecográficos. }\end{array}$ & $\begin{array}{l}\text { A MIC-1/GDF15 foi } \\
\text { positivamente } \\
\text { correlacionada com } \\
\text { CRP, ALT e HDL } \\
\text { alterados quando } \\
\text { crianças com peso } \\
\text { normal e obesas foram } \\
\text { analisadas juntas. }\end{array}$ & $\begin{array}{l}\text { Não foram observadas } \\
\text { diferenças significativas } \\
\text { entre o grupo de crianças } \\
\text { obesas e o grupo controle, } \\
\text { porém os níveis da MIC- } \\
\text { 1/GDF15 tendem a ser } \\
\text { mais elevados em obesos. }\end{array}$ \\
\hline
\end{tabular}

\section{DISCUSSÃO}

Nas últimas décadas, houve um crescimento mundial dos quadros de obesidade entre crianças e adolescentes, tornando esse quadro uma questão de saúde pública (LINHARES, 2016). Nessa perspectiva, os impactos na saúde da obesidade juvenil, como o desenvolvimento de doenças cardiovasculares, tornamse alvo de preocupação crescente por parte de pesquisadores (LEVENSON, 2016). Nesse contexto, a prevenção e o tratamento da obesidade infantil e da dislipidemia assumem caráter multifatorial, associandose, principalmente, a hábitos alimentares e sedentarismo (REUTER et al., 2018; LINHARES et al., 2016).

$\mathrm{O}$ efeito das práticas alimentares de crianças e adolescentes na progressão da obesidade é abordado por Lind et al., (2019), que analisa o padrão dietético nórdico, caracterizado pelo consumo de peixes e óleos derivados poli-insaturados. Tais estudos revelaram que adultos sob esse padrão de dieta apresentaram melhores biomarcadores cardiovasculares e que crianças também apresentaram benefícios fisiológicos diversos. Esses resultados se associam aos encontrados por Del-RíoNavarro et al., (2019), que verificaram que crianças e adolescentes suplementados com ômega 3 apresentaram redução significativa nos índices séricos de triglicerídeos, comparado com o grupo tratado com placebo.

A importância da dieta na redução de biomarcadores atrelados à obesidade infantil também é abordada por Olivares et al., (2015), que investigaram os efeitos do uso de leite desnatado, fermentado e enriquecido com esteróis vegetais sobre pacientes pediátricos. Esse estudo demonstra que as crianças que ingeriram o leite desnatado apresentaram redução dos triglicerídeos basais. Os resultados do estudo de Olivares et al., (2015) se assemelham aos encontrados por Lind et al., (2019), demonstrando que as práticas alimentares durantes as primeiras fases da vida apresentam influência no desenvolvimento de distúrbios metabólicos associados à obesidade.

Em adição aos impactos nutricionais da dieta sobre o metabolismo lipídico de crianças e adolescentes, como a suplementação à base de óleos poli-insaturados - citada por Del-Río-Navarro et. al (2015) - Reuter et. al (2018) busca abordar outras repercussões, além alimentação, sobre o desenvolvimento e o desempenho fisiológicos cardiovasculares de crianças e adolescentes, apresentando consonância à temática e às observações de Lind et. al (2019). Sob esse prisma, Reuter et al., (2018) analisa as relações entre dislipidemia, fatores comportamentais e as aptidões cardiorrespiratórias de crianças e adolescentes. Os resultados demonstram que os indivíduos expostos a elevados intervalos de exposição à TV apresentaram baixos níveis de HDL e os indivíduos que tinham um deslocamento sedentário para escola possuíam altos níveis de LDL. Os achados sugerem relações com a presença de sobrepeso e de obesidade e com comportamento sedentário, entre os motivos que contribuem para os altos índices de dislipidemia. As consequências de hábitos sedentários também são analisadas por Almeida et al., (2016), que constataram que crianças com estilo de vida sedentário apresentam aumento dos valores séricos de LDL, colesterol total e triglicerídeos, além do aumento da dobra cutânea tricipital e da circunferência abdominal.

A respeito da análise de biomarcadores para auxiliar na identificação de crianças obesas e prevenção dos riscos desse distúrbio, os estudos de Yuca et al., (2016) estabelecem a relação entre o macrófago inibitório de citocina 1 - também conhecido como fator de diferenciação de crescimento 15 (MIC-1/GDF15) - e o desenvolvimento de processos inflamatórios 
cardiovasculares. Tal marcador sérico estudado exerce funções-chave na regulação de respostas celulares ao estresse, à inflamação e a injúrias agudas. Os resultados desse estudo encontraram HDL em menores concentrações e parede cardíaca ventricular esquerda dilatada entre as crianças obesas, além de concentração média plasmática de MIC-1/GDF15 maior em pacientes pediátricos obesos em relação aos pacientes do grupo controle. Dessa maneira, embora mais estudos sejam necessários, MIC-1/GDF15 torna-se potencial ferramenta de detecção dos riscos cardíacos e intervenção precoce sobre pacientes pediátricos obesos.

Os estudos de Nielsen et al., (2018) pesquisaram a concentração lipídica circulante entre crianças e adolescentes dinamarqueses. Os resultados demonstraram que crianças e adolescentes da população obesa exibiram concentrações mais altas de colesterol total, LDL triglicerídeos e concentrações mais baixas de HDL em comparação ao grupo de crianças com IMC normal. Esses resultados se assemelham aos encontrados por Almeida et al., (2016), que também constataram a elevação desses níveis séricos em pacientes obesos. Com base nisso, percebe-se que crianças e adolescentes obesos tendem a ter mais dislipidemia que a população na mesma idade com o peso normal.

Sobre o tratamento da dislipidemia na infância, os estudos de Levenson et. al., (2017) analisam os impactos da terapia nutricional com refeições de baixa gordura e de baixa carga glicêmica em um grupo de crianças e adolescentes obesos. Os resultados demonstram que, após 6 meses utilizando a dieta, houve uma diminuição no IMC, da circunferência abdominal, do percentual de gordura corporal e da pressão arterial sistólica. O uso da terapia nutricional também é abordado por Del-Rio-Navarro et.al., (2019) que apontam a suplementação com ômega-3 como uma opção segura e efetiva para o tratamento da hipertrigliceridemia em crianças e adolescentes obesas. Tais resultados se assemelham aos encontrados por Lind et al., (2019) em relação aos benefícios do controle alimentar no combate à dislipidemia.

O tratamento com estatinas em crianças e adolescentes também tem sido alvo de estudos clínicos para os casos de hipercolesterolemia familiar $(\mathrm{HeFH})$. O estudo de Langslet et. al., (2016), demonstraram eficácia e segurança no uso da atovastatina nas doses de
5-40 mg durante 3 anos em crianças e adolescentes com $\mathrm{HeFH}$, tendo sido observado alguns eventos adversos. Entretanto, essa classe de medicamentos, bem como os fibratos e a niacina são indicados apenas para pacientes com hipertrigliceridemia familiar, sendo necessárias outras alternativas terapêuticas para as demais causas de hipertrigliceridemia na infância. (DEL-RÍONAVARRO et al., 2019).

Em relação à realização de cirurgia bariátrica em crianças, a pesquisa de Inge et.al., (2017) acompanhou 57 pacientes que realizaram o procedimento e obteve como resultado uma redução considerável de peso corporal, bem como diminuição na incidência de diabetes mellitus do tipo 2 e hipertensão arterial. Entretanto, mais da metade dos indivíduos ainda possuíam obesidade tipo 2 ou 3 ao fim do estudo, indicando que é necessário associar o tratamento cirúrgico a alterações da dieta e de estilo de vida a longo prazo.

Estudos de Edberg et al., (2019) analisaram o impacto na redução do IMC de crianças e adolescentes submetidas a um programa de incentivo financeiro isoladamente e junto com seus pais. Os jovens do grupo de incentivo isolado recebiam o retorno financeiro integral, enquanto os do grupo de incentivo partilhado dividiam o valor com seus pais. A avaliação da influência de hábitos familiares nas taxas de IMC dos jovens também é descrita por Almeida et al., (2016), que encontraram maiores taxas de triglicerídeos e IMC entre crianças cujos pais também apresentavam elevação desses parâmetros.

Edberg et al., (2019) demonstraram que os jovens do grupo de incentivo isolado apresentaram maior redução do IMC nos três primeiros meses de estudos, em comparação àqueles do grupo de incentivo partilhado. Essa redução poderia ser explicada pelo fato desses jovens terem acesso integral ao valor do incentivo, sendo estimulados a adquirirem hábitos de vida mais saudáveis. Contudo, nos meses seguintes do estudo, o grupo de incentivo partilhado conseguiu alcançar e manter menores níveis de IMC, enquanto o grupo de incentivo isolado teve aumento desse índice. Esses resultados se assemelham aos encontrados por Dunton (2018) à medida que demonstram que a divisão de incentivo com os pais altera o perfil temporal do impacto no IMC, podendo ajudar a sustentar a adoção de comportamentos saudáveis. 


\section{- re revisto

Figura 2. Síntese de Condutas para Tratamento e Prevenção de Obesidade e Dislipidemia na Infância e Adolescência

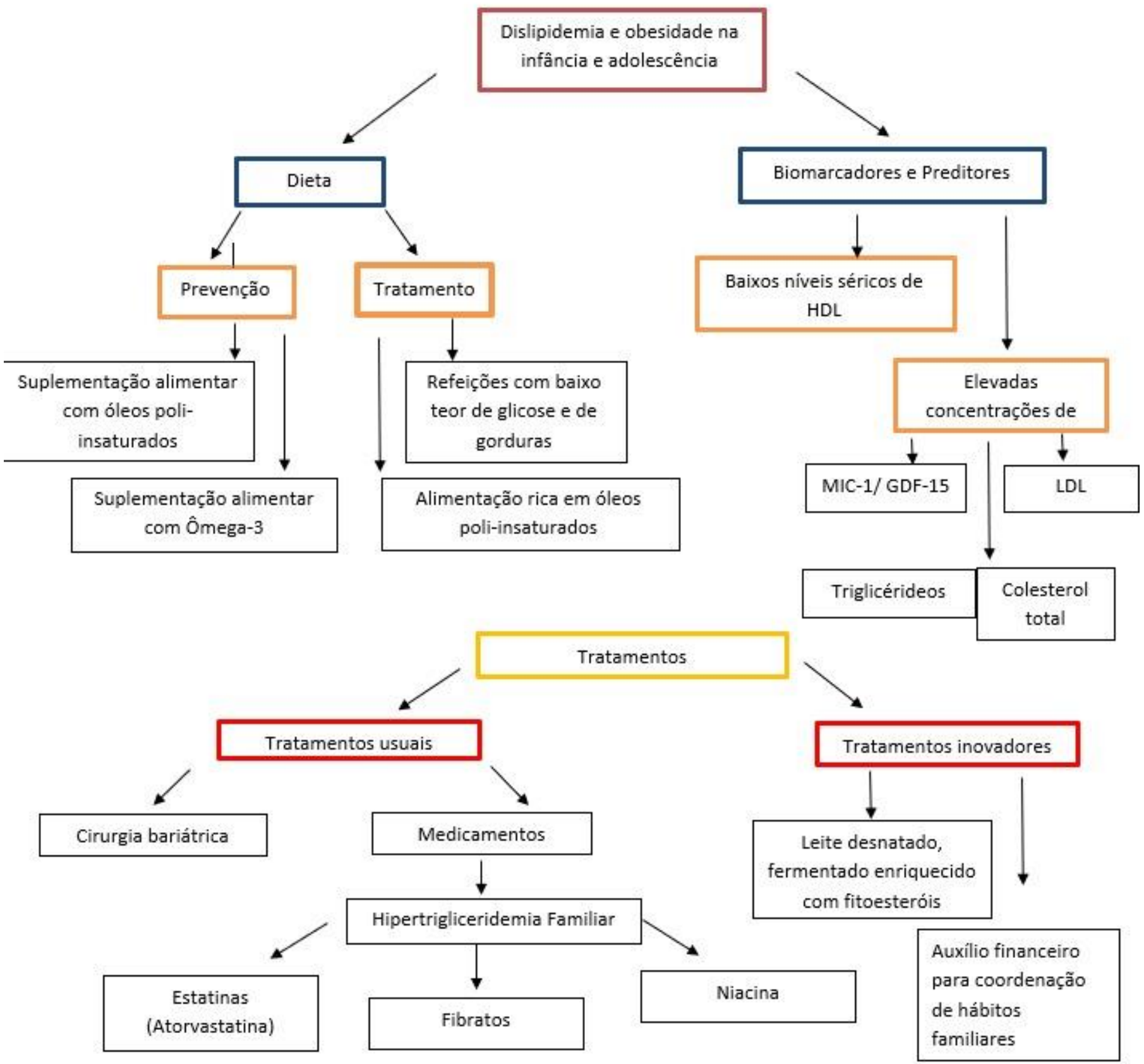

\section{CONCLUSÃO}

Diante do panorama de obesidade na fase infanto-juvenil e das diferentes abordagens na sua profilaxia e tratamento que foi discutida ao longo dessa revisão integrativa, foi possível observar que esse distúrbio metabólico sofre significativa influência do meio, incluindo dieta e sedentarismo, interferindo negativamente sobre a aptidão cardiorrespiratória e desenvolvimento de problemas cardiovasculares.

Foi notada uma redução nos níveis de triglicerídeos basais nos pacientes obesos dos estudos em que houve adição de ômega 3, peixes e óleos poliinsaturados na dieta, mostrando que a adoção de uma 
terapia nutricional é eficiente no tratamento desse distúrbio metabólico. Entretanto, percebeu-se que, em longo prazo, essa terapia vai perdendo eficácia e o incentivo familiar e profissional são necessários na sua continuidade.

Em relação à medicação, o uso de atorvastatina por mais de 3 anos em crianças com hipercolesterolemia familiar demonstrou segurança e eficácia. Os biomarcadores também assumem importante papel para identificação precoce de problemas cardiovasculares consequentes à obesidade, como exemplo se tem o MIC-1/GDF15 que pode ser utilizado como preditor de processos inflamatórios. Ademais, foi observado que a terapia farmacológica, isoladamente, não apresenta benefícios na redução do IMC em pacientes obesos, mas sua associação com a terapia nutricional e mudança no estilo de vida possui uma significativa eficácia.

Portanto, apesar dos resultados benéficos associados à mudança de estilo de vida e uso de terapia farmacológica no tratamento da obesidade e dislipidemia na infância e na adolescência, fazem-se necessários novos estudos sobre a segurança e eficácia em longo prazo dessa associação terapêutica.

\section{REFERÊNCIAS}

ALCÂNTARA NETO, Osvaldo Dário de; SILVA, Rita de Cássia Ribeiro; ASSIS, Ana Marlúcia Oliveira; PINTO, Elizabeth de Jesus. Fatores associados à dislipidemia em crianças e adolescentes de escolas públicas de Salvador, Bahia. Revista Brasileira de Epidemiologia, [S.L.], v. 15, n. 2, p. 335-345, jun. 2012. FapUNIFESP (SciELO). http://dx.doi.org/10.1590/s1415-790x2012000200011.

ALMEIDA, Patrícia Casagrande Dias de; SILVA, Janine Pereira da; PINASCO, Gustavo Carreiro; HEGNER, Christina Cruz; MATTOS, Danielle Cabrini; POTRATZ, Mateus Oliveira; BRAVIN, Lucas Santos; SILVA, Valmin Ramos; LAMOUNIER, Joel Alves. Lipid profile in schoolchildren in Vitória - Brazil. Journal Of Human Growth And Development, [S.L.], v. 26, n. 1, p. 61, 28 abr. 2016.

NEPAS.

http://dx.doi.org/10.7322/jhgd.110989.

CAMARGOS, Ana Cristina Resende; AZEVEDO, Bárbara Nayara Souza; SILVA, Darlene da; MENDONÇA, Vanessa Amaral; LACERDA, Ana Cristina Rodrigues. Prevalência de sobrepeso e de obesidade no primeiro ano de vida nas Estratégias Saúde da Família. Cadernos Saúde Coletiva, [S.L.], v. 27, n. 1, p. 32-38, mar. 2019.UNIFESP (SciELO). http://dx.doi.org/10.1590/1414-462x201900010010.

DANIELS, Stephen; GREER, Frank.. Lipid Screening and Cardiovascular Health in Childhood. Pediatrics, [S.L.], v. 122, n. 1, p. 198-208, 1 jul. 2008. American Academy of Pediatrics (AAP). http://dx.doi.org/10.1542/peds.2008-1349.

DEL-RÍO-NAVARRO, Blanca; MIRANDA-LORA, América; HUANG, Fengyang; HALL-MONDRAGON,
Margareth; LEIJA-MARTÍNEZ, José. Effect of supplementation with omega-3 fatty acids on hypertriglyceridemia in pediatric patients with obesity. Journal of Pediatric Endocrinology and Metabolism, [S.L.], v. 32, n. 8, p. 811-819, 27 ago. 2019. Walter de Gruyter GmbH. http://dx.doi.org/10.1515/jpem-20180409.

DUNTON, Genevieve. Sustaining Health-Protective Behaviors Such as Physical Activity and Healthy Eating. Jama, [S.L.], v. 320, n. 7, p. 639, 21 ago. 2018. American Medical Association

(AMA). http://dx.doi.org/10.1001/jama.2018.6621

EDBERG, Dana; MUKHOPADHYAY, Sankar; WENDEL, Jeanne. Incentive design to boost health for juveniles with Medicaid coverage: evidence from a field experiment. Economics \& Human Biology, [S.L.], v. 33, p. 101-115, maio 2019. Elsevier BV. http://dx.doi.org/10.1016/j.ehb.2019.01.002.

PEDIATRICS, American Academy Of. Expert Panel on Integrated Guidelines for Cardiovascular Health and Risk Reduction in Children and Adolescents: summary report. Pediatrics, [S.L.], v. 128, n. , p. 213-256, 14 nov. 2011. American Academy of Pediatrics (AAP). http://dx.doi.org/10.1542/peds.2009-2107c

FRANCA, Everaldo de; ALVES, João Guilherme Bezerra. Dislipidemia entre crianças e adolescentes de Pernambuco. Arquivos Brasileiros de Cardiologia, [S.L.], v. 87, n. 6, p. 722-727, dez. 2006. FapUNIFESP (SciELO). http://dx.doi.org/10.1590/s0066782x2006001900007.

GAMA, Sueli Rosa; CARVALHO, Marilia Sá; 
CHAVES, Célia Regina Moutinho de Miranda. Prevalência em crianças de fatores de risco para as doenças cardiovasculares. Cadernos de Saúde Pública, [S.L.], v. 23, n. 9, p. 2239-2245, set. 2007. FapUNIFESP (SciELO). $311 \times 2007000900032$.

http://dx.doi.org/10.1590/s0102-

GIDDING, Samuel; KEITH, Scott; FALKNER, Bonita. Adolescent and adult African Americans have similar metabolic dyslipidemia. Journal Of Clinical Lipidology, [S.L.], v. 9, n. 3, p. 368-376, maio 2015. Elsevier BV. http://dx.doi.org/10.1016/j.jacl.2014.11.010.

GIULIANO, Isabela de Carlos Back; COUTINHO, Mário Sérgio Soares de Azeredo; FREITAS, Sérgio Fernando Torres de; PIRES, Maria Marlene de Souza; ZUNINO, João Nilson; RIBEIRO, Robespierre Queiroz da Costa. Lípides séricos em crianças e adolescentes de Florianópolis, SC: estudo floripa saudável 2040. Arquivos Brasileiros de Cardiologia, [S.L.], v. 85, n. 2, p. 1-6, ago. 2005. FapUNIFESP (SciELO). http://dx.doi.org/10.1590/s0066-782x2005001500003.

HIGGINS, Victoria; ADELI, Khosrow. Pediatric decision limits for lipid parameters in the Brazilian population. Jornal de Pediatria, [S.L.], v. 95, n. 2, p. 124 127, mar. 2019. Elsevier BV. http://dx.doi.org/10.1016/j.jped.2018.05.002.

INGE, Thomas; JENKINS, Todd; A XANTHAKOS, Stavra; DIXON, John; DANIELS, Stephen; ZELLER, Meg; A HELMRATH, Michael. Long-term outcomes of bariatric surgery in adolescents with severe obesity (FABS-5+): a prospective follow-up analysis. The Lancet Diabetes \& Endocrinology, [S.L.], v. 5, n. 3, p. 165-173, mar. 2017. Elsevier BV. http://dx.doi.org/10.1016/s2213-8587(16)30315-1.

JANSSEN, Ian; KATZMARZYK, Peter; SRINIVASAN, Sathanur; CHEN, Wei; MALINA, Robert; BOUCHARD, Claude; BERENSON, Gerald. Utility of Childhood BMI in the Prediction of Adulthood Disease: comparison of national and international references. Obesity Research, [S.L.], v. 13, n. 6, p. 11061115, jun. $2005 . \quad$ Wiley. http://dx.doi.org/10.1038/oby.2005.129.

JOYCE, Nina; WELLENIUS, Gregory A.; DORE, David D.; NEWBURGER, Jane W.; ZACHARIAH, Justin P.. Patterns of Lipid Lowering Therapy among Children Ages 8-20 Years. The Journal Of Pediatrics, [S.L.], v. 167, n. 1, p. 113-119, jul. 2015. Elsevier BV. http://dx.doi.org/10.1016/j.jpeds.2015.04.004.
LANGSLET, Gisle; BREAZNA, Andrei; DROGARI, Euridiki. A 3-year study of atorvastatin in children and adolescents with heterozygous familial hypercholesterolemia. Journal Of Clinical Lipidology, [S.L.], v. 10, n. 5, p. 1153-1162, set. 2016. Elsevier BV. http://dx.doi.org/10.1016/j.jacl.2016.05.010.

LEVENSON, Amy; MILLIREN, Carly; BIDDINGER, Sudha; EBBELING, Cara; FELDMAN, Henry; LUDWIG, David; FERRANTI, Sarah de Calorically restricted diets decrease PCSK9 in overweight adolescents. Nutrition, Metabolism And Cardiovascular Diseases, [S.L.], v. 27, n. 4, p. 342-349, abr. 2017. Elsevier

BV. http://dx.doi.org/10.1016/j.numecd.2016.12.010.

LIND, Torbjörn; JOHANSSON, Ulrica; ÖHLUND, Inger; LINDBERG, Lene; LÖNNERDAL, Bo; TENNEFORS, Catharina; HERNELL, Olle. Study protocol: optimized complementary feeding study (otis). Bmc Public Health, [S.L.], v. 19, n. 1, p. 1-11, 31 jan. 2019. Springer Science and Business Media LLC. http://dx.doi.org/10.1186/s12889-019-6466-1.

LINHARES, Francisca Michelli Medeiros. Obesidade infantil: influência dos pais sobre a alimentação e estilo de vida

dos filhos. Temas em Saúde, Patos, p. 460-481, 2016. $\mathrm{http}: / /$ temasemsaude.com/wpcontent/uploads/2016/08/16226.pdf

MILLER, Michael; STONE, Neil; BALLANTYNE, Christie; BITTNER, Vera; CRIQUI, Michael; GINSBERG, Henry; GOLDBERG, Anne Carol; HOWARD, William James; JACOBSON, Marc; KRISETHERTON, Penny. Triglycerides and Cardiovascular Disease. Circulation, [S.L.], v. 123, n. 20, p. 2292-2333, 24 maio 2011. Ovid Technologies (Wolters Kluwer Health).

http://dx.doi.org/10.1161/cir.0b013e3182160726.

NIELSEN, Tenna Ruest Haarmark; LAUSTENTHOMSEN, Ulrik; FONVIG, Cilius Esmann; BØJSØE, Christine; PEDERSEN, Lise; BRATHOLM, Palle Skov; HANSEN, Torben; PEDERSEN, Oluf; HOLM, JensChristian. Dyslipidemia and reference values for fasting plasma lipid concentrations in Danish/North-European White children and adolescents. Bmc Pediatrics, [S.L.], v. 17, n. 1, p. 1-11, 28 abr. 2017. Springer Science and Business Media LLC. http://dx.doi.org/10.1186/s12887017-0868-y.

OLIVARES, Javier Andrés Blumenfeld. LECHE ENRIQUECIDA CON ESTEROLES VEGETALES 
COMO ESTRATEGIA PARA. Nutricion Hospitalaria, [S.L.], n. 3, p. 1056-1060, 1 set. 2015. http://dx.doi.org/10.3305/nh.2015.32.3.9319.

OLIVEIRA, Carla Braga Campelo de; BRITO, Lorena Almeida; FREITAS, Morgana Andrade; SOUZA, Marcos Porto Arrais de; RêGO, Juliana Magalhães da Cunha; MACHADO, Richele Janaína de Araújo. Obesidade: inflamação e compostos bioativos. Journal Of Health \& Biological Sciences, [S.L.], v. 8, n. 1, p. 15, 3 jan. 2020. http://dx.doi.org/10.12662/23173076jhbs.v8i1.2785.p1-5.2020.

PASTOR-VILLAESCUSA, Belén; CABALLEROVILLARRASO, Javier; CAÑETE, M. Dolores; HOYOS, Raúl; MALDONADO, José; BUENO, Gloria; LEIS, Rosaura; GIL, Ángel; CAÑETE, Ramón; AGUILERA, Concepción M.. Evaluation of differential effects of metformin treatment in obese children according to pubertal stage and genetic variations: study protocol for a randomized controlled trial. Trials, [S.L.], v. 17, n. 1, p. 1-10, 18 jul. 2016. Springer Science and Business Media LLC. http://dx.doi.org/10.1186/s13063016-1403-4.

REUTER, Cézane Priscila; BRAND, Caroline; SILVA, Priscila Tatiana da; REUTER, Éboni Marília; RENNER, Jane Dagmar Pollo; FRANKE, Silvia Isabel Rech; MELLO, Elza Daniel de; BURGOS, Leandro Tibiriçá; SCHNEIDERS, Letícia de Borba; BURGOS, Miria Suzana. Relationship between Dyslipidemia, Cultural Factors, and Cardiorespiratory Fitness in Schoolchildren. Arquivos Brasileiros de Cardiologia, [S.L.], v. 6, n. 112, p. 729-736, 2019. http://dx.doi.org/10.5935/abc.20190068.

YUCA, Sevil Ari; CIMBEK, Emine Ayça Cimbek; ŞEN, Yaşar; GÜVENÇ, Osman; VATANSEV, Husamettin; BUğRUL, Fuat; GÜN, Feryal; ORAN, Bülent. The Relationship between Metabolic Parameters, Cardiac Parameters and MIC-1/GDF15 in Obese Children. Experimental and Clinical Endocrinology \& Diabetes, [S.L.], v. 125, n. 02, p. 86-90, 17 out. 2016. Georg Thieme Verlag KG. http://dx.doi.org/10.1055/s-0042114220 . 